\title{
Uplink achievable rate in underlay random access OFDM-based cognitive radio networks
}

\author{
Han-Bae Kong ${ }^{{ }^{*}}$ (D), Sabit Ekin² ${ }^{2}$ Erchin Serpedin ${ }^{1}$ and Khalid A. Qaraqe ${ }^{3}$
}

\begin{abstract}
This paper investigates the uplink achievable rate of secondary users (SUs) in underlay orthogonal frequency division multiplexing based cognitive radio networks, where the SUs randomly access the subcarriers of the primary network. In practice, the primary base stations (PBSs), such as cellular base stations, may not be placed close to each other to mitigate the interferences among them. In this regard, we model the spatial distribution of the PBSs as a $\beta$-Ginibre point process which captures the repulsive placement of the PBSs. It is assumed that in order to alleviate the interferences at the PBSs from the SUs, each SU controls its transmit power based on the average interference level at the closest PBS induced by the SU. We first analytically identify the characteristics of the transmit powers at the SUs. Then, tight approximations of the uplink achievable rate of the secondary network are provided in two different scenarios that assume either a decentralized or centralized allocation of the SUs' subcarriers, respectively. The accuracy of our analytical results is validated by simulation results.
\end{abstract}

Keywords: Orthogonal frequency division multiplexing, Cognitive radio, Random access, Underlay network, Stochastic geometry

\section{Introduction}

Orthogonal frequency division multiplexing (OFDM)based cognitive radios have been recognized as a promising solution to overcome the radio frequency spectrum scarcity [1]. In cognitive radio networks (CRNs), secondary users (SUs) access the spectrum in general via three different approaches, i.e., interweave, overlay, and underlay, respectively. Among the access models, due to their simplicity, underlay CRNs, where SUs transmit their data without spectrum sensing as long as the interference level at the primary network (PN) remains acceptable, have been widely explored [1-3].

During the past decade, many researchers have studied underlay OFDM-based CRNs where the locations of nodes in the overlapping networks are deterministic and known [4-8]. The work [4] investigated the average $\mathrm{SU}$ achievable rate when random subcarrier allocation schemes are adopted at the secondary network (SN), and

\footnotetext{
*Correspondence: hbkong@tamu.edu

${ }^{1}$ Department of Electrical and Computer Engineering, Texas A\&M University, College Station 77843, TX, USA

Full list of author information is available at the end of the article
}

the number of allocated subcarriers for users is fixed. The number of subcarrier collisions between the PN and the SN was analyzed in [5] assuming fixed and random numbers of subcarriers at each user. The authors in [6] proposed a joint subcarrier pairing and power allocation method to maximize the SU's throughput in relayaided OFDM-based CRNs. The problem of determining the power and subcarrier allocation levels that maximize the average achievable throughput of multi-user OFDM-based CRNs under the constraint of an allowable interference threshold at the PN was examined in [9] and [10].

Assessing the underlay CRN performance by taking into account the spatial distributions of CRN nodes as point processes has attracted much attention recently [11]. In [12], the outage probability of an underlay CRN was established under the assumption that the locations of primary base stations (PBSs) and secondary base stations (SBSs) are modeled as two independent Poisson point processes (PPPs). Also, $[13,14]$ studied the coverage probability of the uplink transmission of an underlay CRN in which the distributions of PBSs, SBSs, primary users (PUs), and 
SUs follow independent PPPs. However, to the best of our knowledge, the performance of an underlay OFDMbased CRN, which depends implicitly on the employed subcarrier allocation method, has not been addressed yet.

Due to its mathematical tractability, the PPP whose points are independent has been widely adopted to model various types of wireless networks where nodes are placed in an unplanned fashion [15]. However, when an operator designs a network, transmitters in the network may not be placed in close proximity to each other. This measure is to reduce the interferences among the transmitters and to extend the coverage region, and it incurs a repulsive feature in the networks $[16,17]$. The $\beta$-Ginibre point process (GPP) [18] is a repulsive point process which can reflect the repulsive nature and presents the additional benefit of including the PPP as a particular distribution [19]. For this reason, the $\beta$-GPP has been applied to analyze the coverage probabilities of single-tier and multi-tier downlink cellular networks in [20] and [21], respectively. Despite the fact that PBSs in CRNs may exhibit a repulsive behavior, the performance of an underlay OFDMbased CRN which considers the repulsion has not been investigated yet.

\section{Method}

This paper examines the performance of an underlay OFDM-based CRN that assumes multiple PBSs, SBSs, PUs, and SUs, and a power control method in the SN to mitigate the interferences at PBSs from the SUs. We study the SU uplink achievable rate under two scenarios: with and without a centralized allocation of subcarriers at the SN, respectively, and which affect differently the collisions among the SUs subcarriers. The PNs can stand for various networks with a repulsive feature such as cellular networks or wireless sensor networks, and hence the locations of the PBSs are modeled as a $\beta$-GPP. On the other hand, since the SBSs in a SN can represent individual users or devices deployed by multiple different operators/users, the repulsion among the positions of the SBSs is negligible, and thus the spatial distribution of the SBSs is assumed to follow a PPP.

The contributions of this paper are summarized next:

- First, we present the expressions of the cumulative distribution function (CDF) and probability density function (PDF) of transmit powers at SUs when the positions of the PBSs are modeled as a $\beta$-GPP.

- Then, we derive approximations of the average of the SU uplink achievable rate for the cases where the SUs subcarriers are allocated in a decentralized or centralized manner at the SN.

- Lastly, we explore the impact of subcarrier collisions among the SUs and repulsion among the locations of PBSs on the SU achievable rate.
The organization of this paper is as follows: Section 3 describes the system model of an underlay OFDM-based CRN. The characteristics of the transmit powers at SUs and the SU uplink achievable rate are investigated in Section 4. In Section 5, numerical simulation results are provided to validate the tightness of the derived analytical results. Lastly, conclusions are made in Section 6.

The following notations are used throughout the paper. $\mathbb{P}(A)$ and $\mathbb{E}[X]$ stand for the probability of an event $A$ and the mean of a random variable $X$, respectively. The operators $\backslash$ and $\|\cdot\|$ denote the set difference operation and the Euclidean 2-norm, respectively. The list of symbols used in this paper and their definitions is provided in Table 1.

\section{System model}

We consider an underlay OFDM-based CRN consisting of a PN and a SN. We will focus on assessing the uplink performance of the SN when each SU (or PU) is associated with the SBS (or PBS) providing the highest average channel gain, i.e., the nearest SBS (or PBS), and transmits its data to the associated SBS (or PBS).

To capture the repulsive behavior among the locations of PBSs, the spatial distribution of the PBSs is modeled as a $\beta$-GPP $\Phi_{P}$ with repulsion parameter $\beta$ and intensity $\lambda_{P}$. Here, the parameter $\beta \in(0,1]$ determines the degree of repulsion among the positions of the PBSs. More specifically, the PBSs are more evenly distributed when $\beta$ increases and $\Phi_{P}$ converges to a homogeneous PPP with intensity $\lambda_{P}$ when $\beta \rightarrow 0$. The locations of SBSs are

Table 1 List of notations

\begin{tabular}{|c|c|}
\hline Notation & Definition \\
\hline$\Phi_{P}$ & $\begin{array}{l}\beta \text {-GPP with repulsion parameter } \beta \text { and intensity } \lambda_{P} \\
\text { which represents the locations of PBSs }\end{array}$ \\
\hline$\Phi_{S}$ & $\begin{array}{l}\text { PPP with intensity } \lambda_{s} \text { which represents the locations } \\
\text { of SBSs }\end{array}$ \\
\hline$\Phi_{P}^{(u)}\left(\Phi_{S}^{(u)}\right)$ & $\begin{array}{l}\text { PPP with intensity } \lambda_{\rho}^{(u)}\left(\lambda_{S}^{(u)}\right) \text { which represents the } \\
\text { locations of PUs (SUs) }\end{array}$ \\
\hline$N$ & Number of available subcarriers \\
\hline$N_{p}\left(N_{S}\right)$ & Number of subcarriers for each PU (SU) \\
\hline$\tau$ & Pre-defined interference threshold at PBSs \\
\hline$\alpha_{P}\left(\alpha_{S}\right)$ & $\begin{array}{l}\text { Path loss exponent for channels between users and } \\
\text { PBSs (SBSS) }\end{array}$ \\
\hline$P_{S, \mathbf{x}}$ & Transmit power at the SU at $\mathbf{x}$ \\
\hline$P_{P}$ & Transmit power at the PUs \\
\hline$h_{\mathbf{x}, i}\left(g_{\mathbf{x}, i}\right)$ & $\begin{array}{l}\text { Power of small-scale fading channel between the } \\
\text { tagged SBS and the SU (PU) at } \mathbf{x} \text { for the ith subcarrier }\end{array}$ \\
\hline$\sigma^{2}$ & Power of additive white Gaussian noise (AWGN) \\
\hline$\gamma_{i}$ & $\begin{array}{l}\text { Signal-to-interference-plus-noise ratio (SINR) for the } \\
\text { ith subcarrier }\end{array}$ \\
\hline C & Instantaneous SU achievable rate \\
\hline$\hat{C}$ & Average SU achievable rate \\
\hline
\end{tabular}


assumed to follow a homogeneous PPP $\Phi_{S}$ with intensity $\lambda_{S}$. Also, we model the distributions of PUs and SUs as a homogeneous PPP $\Phi_{P}^{(u)}$ with intensity $\lambda_{P}^{(u)}$ and a homogeneous PPP $\Phi_{S}^{(u)}$ with intensity $\lambda_{S}^{(u)}$, respectively. It is presumed that $\Phi_{P}, \Phi_{S}, \Phi_{P}^{(u)}$, and $\Phi_{S}^{(u)}$ are mutually independent.

We assume that there are $N$ available subcarriers, and the number of allocated subcarriers for each PU and each $\mathrm{SU}$ is $N_{P}$ and $N_{S}$, respectively ${ }^{1}$. In the PN, to alleviate the interferences among the PUs, each PBS allocates each subcarrier to at most one PU which is associated to the PBS so that the PUs communicating with the same PBS do not collide. More specifically, if the number of PUs in the Voronoi cell of a PBS is $M_{P}$, as each PU requests $N_{P}$ subcarriers, the number of subcarriers requested by the connected PUs is equal to $M_{P} N_{P}$. When $M_{P} N_{P} \leq N$, the allocations of PUs subcarriers are conducted by maintaining orthogonality among them. On the other hand, if $M_{P} N_{P}>N$, each of $N$ subcarriers is assigned to a single PU that is chosen in a random fashion.

Two types of subcarrier allocation methods for the $\mathrm{SN}$ are considered, i.e., decentralized subcarrier allocation (DSA) and centralized subcarrier allocation (CSA). When the DSA is applied, each SU randomly selects $N_{S}$ subcarriers independently with respect to other SUs and PUs. On the other hand, when the CSA is adopted, each SBS sequentially and randomly assigns orthogonal sets of subcarriers to its associated SUs. More precisely, if the number of SUs in the Voronoi cell of a SBS is equal to $M_{S}$ and $M_{S} N_{S} \leq N$, each SU connected to the SBS utilizes $N_{S}$ subcarriers which are orthogonal with other subcarriers allocated for other SUs in the Voronoi cell. Otherwise, if $M_{S} N_{S}>N$, each of $N$ subcarriers is allocated to a single $\mathrm{SU}$ that is selected in a random fashion [4].

Since data transmissions from SUs cause interferences at PBSs, they lead to a degradation in the $\mathrm{PN}$ performance. On the other hand, the $\mathrm{SN}$ performance can be improved with larger transmit powers at the SUs. In this context, it would be reasonable to increase the transmit powers at the SUs as long as the interferences at the PBSs do not exceed a certain threshold. Hence, we consider the scenario where each SU adjusts its transmit power based on the average interference level at the closest PBS induced by the SU. Under this scenario, the transmit power at the SU situated at $\mathbf{x} \in \mathbb{R}^{2}$ is expressed as

$$
P_{S, \mathbf{x}}=\tau r_{P, \mathbf{x}}^{\alpha_{P}},
$$

where

$$
r_{P, \mathbf{x}}=\min _{\mathbf{y} \in \Phi_{P}}\|\mathbf{x}-\mathbf{y}\|,
$$

where $\tau, \alpha_{P}$, and $r_{P, \mathbf{x}}$ represent the pre-defined interference threshold, path loss exponent for channels between SUs and PBSs, and distance between the SU at $\mathbf{x}$ and its nearest PBS, respectively. When the maximum transmit power constraint at SUs is considered, the transmit power at the $\mathrm{SU}$ at $\mathbf{x}$ becomes

$$
P_{S, \mathbf{x}}=\min \left(\tau r_{P, \mathbf{x}}^{\alpha_{P}}, P_{\max }\right),
$$

where $P_{\max }$ is the maximum transmit power allowed at SUs.

Denote now the set of indices of all subcarriers as $\mathcal{N}=\{1, \ldots, N\}$. We consider a typical SU and represent the set of indices of the subcarriers selected by the typical SU by $\mathcal{F}=\left\{f_{1}, \ldots, f_{N_{S}}\right\}$, where $\mathcal{F} \subset \mathcal{N}$. Since the homogeneous PPP is stationary [22], without loss of generality, we assume that the typical $\mathrm{SU}$ is located at the origin $o$. We term the SBS which is associated with the typical SU as tagged $S B S$.

Denoting the location of the tagged SBS by $\mathbf{y} \in \mathbb{R}^{2}$, for the subcarrier $f_{i} \in \mathcal{F}$, the signal-to-interference-plusnoise ratio (SINR) $\gamma_{i}$ is expressed as

$$
\gamma_{i}=\frac{P_{S, o} h_{o, i}\|\mathbf{y}\|^{-\alpha_{S}}}{I_{S, i}+I_{P, i}+\sigma^{2}}
$$

where

$$
\begin{aligned}
& \mathbf{y}=\underset{\mathbf{z} \in \Phi_{S}}{\arg \min }\|\mathbf{z}\|, \\
& I_{S, i} \triangleq \sum_{\mathbf{x} \in \Phi_{S, i}^{(u)} \backslash o} P_{S, \mathbf{x}} h_{\mathbf{x}, i}\|\mathbf{x}-\mathbf{y}\|^{-\alpha_{S},} \\
& I_{P, i} \triangleq \sum_{\mathbf{x} \in \Phi_{P, i}^{(u)}} P_{P} g_{\mathbf{x}, i}\|\mathbf{x}-\mathbf{y}\|^{-\alpha_{S},}
\end{aligned}
$$

where $\sigma^{2}$ indicates the power of additive white Gaussian noise (AWGN), $P_{P}$ denotes the transmit power at PUs and $\alpha_{S}$ is the path loss exponent for channels between users and SBSs. We define $\Phi_{S, i}^{(u)}$ and $\Phi_{P, i}^{(u)}$ as the point processes representing the distributions of the SUs and the PUs employing the subcarrier $f_{i}$, respectively. Also, $h_{\mathbf{x}, i}$ (or $\left.g_{\mathbf{x}, i}\right)$ stands for the power of small-scale fading channel between the tagged SBS and the SU (or PU) at $\mathbf{x}$ for the subcarrier $f_{i}$, which is independent with respect to $\Phi_{P}$, $\Phi_{S}, \Phi_{P}^{(u)}$, and $\Phi_{S}^{(u)}$. It is assumed that the small-scale fading channels are Rayleigh distributed, and hence $\left\{h_{\mathbf{x}, i}\right\}$ and $\left\{g_{\mathbf{x}, i}\right\}$ are independent exponential random variables with unit mean.

Remarkably, even when the CSA is adopted in the SN, there exist interferences from other SUs which are connected to other SBSs, i.e., $I_{S, i}$ in (4). In addition, since both the CSA and the DSA do not consider the subcarrier allocation in the PN, signals from PUs may collide with the signal transmitted at the typical $\mathrm{SU}$, and this collision is quantified by $I_{P, i}$ in (5). 
For the typical SU, the total instantaneous achievable rate is expressed as

$$
C=\sum_{i \in \mathcal{N} \text { s.t. } f_{i} \in \mathcal{F}} \log _{2}\left(1+\gamma_{i}\right)
$$

In the following section, we will derive approximations of the average of $C$ in (6) and confirm the tightness of the approximations in Section 5.

\section{Performance analysis}

\subsection{Characteristics of transmit powers at SUs}

From [20, Prop. 1], for the $\beta$-GPP $\Phi_{P}=\left\{\mathbf{y}_{k}\right\}_{k \in \mathbb{N}}$, the set $\left\{\left\|\mathbf{y}_{k}\right\|^{2}\right\}_{k \in \mathbb{N}}$ follows the same distribution as the set $\Xi_{P}$ constructed from a sequence $B_{P, i} \sim \mathcal{G}\left(i, \beta /\left(\pi \lambda_{P}\right)\right)$ of independent random variables by deleting each $B_{P, i}$ independently and with probability $1-\beta$, where $\mathcal{G}(a, b)$ denotes a gamma random variable with shape parameter $a$ and scale parameter $b$. As the $\beta$-GPP is stationary [18], the characteristics of $P_{S, \mathbf{x}}$ are invariant to the location $\mathbf{x}$. Thus, for brevity of presentation, we omit the subscript x. We introduce the CDF and PDF of transmit power $P_{S}$ in (1) in the following lemma.

\section{Lemma 1 The $C D F$ of $P_{S}$ in (1) is expressed as}

$$
F_{P_{S}}(x)=1-\prod_{k=1}^{\infty}\left(1-\frac{\beta}{\Gamma(k)} \gamma\left(k, \frac{\pi \lambda_{P} x^{2 / \alpha_{P}}}{\beta \tau^{2 / \alpha_{P}}}\right)\right),
$$

where $\gamma(a, b)=\int_{0}^{b} t^{a-1} e^{-t} d t$ and $\Gamma(a)=\int_{0}^{\infty} t^{a-1} e^{-t} d t$ denote the lower incomplete gamma function and gamma function, respectively. The PDF of $P_{S}$ is given by

$$
\begin{aligned}
f_{P_{S}}(x)= & \frac{2 \beta}{\alpha_{P}} \exp \left(-\frac{\pi \lambda_{P}}{\beta}(x / \tau)^{2 / \alpha_{P}}\right) \prod_{k=1}^{\infty} a_{k}(x) \\
& \times \sum_{k=1}^{\infty} \frac{1}{a_{k}(x) \Gamma(k)}\left(\frac{\pi \lambda_{P}}{\beta \tau^{2 / \alpha_{P}}}\right)^{k} x^{2 k / \alpha_{P}-1},
\end{aligned}
$$

where $a_{k}(x) \triangleq 1-\frac{\beta}{\Gamma(k)} \gamma\left(k, \pi \lambda_{P} x^{2 / \alpha_{P}} /\left(\beta \tau^{2 / \alpha_{P}}\right)\right)$.

\section{Proof See Appendix A.}

From the expression (7), we can see that the CDF of $P_{S}$ is an increasing (or a decreasing) function of $\lambda_{P}$ (or $\tau$ ), and this indicates that the transmit power $P_{S}$ gets smaller as $\lambda_{P}$ grows and $\tau$ decays. When the maximum available transmit power is considered for SUs, the CDF of $P_{S}$ in (2) is represented as

$$
\begin{aligned}
& F_{P_{S}}(x) \\
& = \begin{cases}1-\prod_{k=1}^{\infty}\left(1-\frac{\beta}{\Gamma(k)} \gamma\left(k, \frac{\pi \lambda_{P} x^{2 / \alpha_{P}}}{\beta \tau^{2 / \alpha_{P}}}\right)\right) & \text { if } x<P_{\max }, \\
1 & \text { otherwise, }\end{cases}
\end{aligned}
$$

and the corresponding PDF is given by

$$
\begin{aligned}
f_{P_{S}}(x)= & \frac{2 \beta}{\alpha_{P}} \exp \left(-\frac{\pi \lambda_{P}}{\beta}(x / \tau)^{2 / \alpha_{P}}\right) \prod_{k=1}^{\infty} a_{k}(x) \\
& \times \sum_{k=1}^{\infty} \frac{1}{a_{k}(x) \Gamma(k)}\left(\frac{\pi \lambda_{P}}{\beta \tau^{2 / \alpha_{P}}}\right)^{k} x^{2 k / \alpha_{P}-1} \mathbb{1}\left(x<P_{\max }\right) \\
& +\mathbb{1}\left(x=P_{\max }\right) \varphi
\end{aligned}
$$

where $\varphi=\prod_{k=1}^{\infty}\left(1-\frac{\beta}{\Gamma(k)} \gamma\left(k, \frac{\pi \lambda_{P} P_{\max }^{2 / \alpha_{P}}}{\beta \tau^{2 / \alpha_{P}}}\right)\right)$ and $\mathbb{1}(\cdot)$ is the indicator function.

Now, let us look at the case where the spatial distribution of the PBSs follows a homogeneous PPP with intensity $\lambda_{P}$, i.e., $\beta \rightarrow 0$. Note that for a sufficiently small positive value $\omega$ and for $\delta>0,-(1+\delta) \omega \leq$ $\ln (1-\omega) \leq-\omega$. Let us define $\bar{a}_{k}(x) \triangleq 1-a_{k}(x)=$ $\frac{\beta}{\Gamma(k)} \gamma\left(k, \pi \lambda_{P} x^{2 / \alpha_{P}} /\left(\beta \tau^{2 / \alpha_{P}}\right)\right)$. Then, since $\bar{a}_{k}(x) \rightarrow 0$ as $\beta \rightarrow 0$ for all $k \in \mathbb{N}$, when $\beta \rightarrow 0$, we get

$$
\begin{aligned}
& -(1+\delta) \bar{a}_{k}(x) \leq \ln \left(1-\bar{a}_{k}(x)\right) \leq-\bar{a}_{k}(x) \\
& \Leftrightarrow \exp \left(-(1+\delta) \sum_{k=1}^{\infty} \bar{a}_{k}(x)\right) \leq \prod_{k=1}^{\infty}\left(1-\bar{a}_{k}(x)\right) \\
& \quad \leq \exp \left(-\sum_{k=1}^{\infty} \bar{a}_{k}(x)\right) .
\end{aligned}
$$

By letting $\delta \rightarrow 0$, we have

$$
\begin{aligned}
F_{P_{S}}(x) & =1-\prod_{k=1}^{\infty}\left(1-\bar{a}_{k}(x)\right) \\
\underset{\beta \rightarrow 0}{\longrightarrow} & 1-\exp \left(-\sum_{k=1}^{\infty} \bar{a}_{k}(x)\right) \\
& =1-\exp \left(-\beta \int_{0}^{\frac{\pi \lambda_{P} x^{2 / \alpha_{P}}}{\beta \tau^{2 / \alpha_{P}}}} \sum_{k=1}^{\infty} \frac{t^{k-1}}{\Gamma(k)} e^{-t} d t\right) \\
& =1-\exp \left(-\frac{\pi \lambda_{P} x^{2 / \alpha_{P}}}{\tau^{2 / \alpha_{P}}}\right),
\end{aligned}
$$

which is the same with the result for the homogeneous PPP [22]. In addition, by differentiating $F_{P_{S}}(x)$ with respect to $x$, the PDF of $P_{S}$ is given by

$$
f_{P_{S}}(x)=\frac{2 \pi \lambda_{P} x^{2 / \alpha_{P}-1}}{\alpha_{P} \tau^{2 / \alpha_{P}}} \exp \left(-\frac{\pi \lambda_{P} x^{2 / \alpha_{P}}}{\tau^{2 / \alpha_{P}}}\right) .
$$

Each PU may adjust its transmit power based on the distance between the PU and its associated PBS. In this case, the CDF and PDF of transmit powers at PUs can be derived in the same manner as in Lemma 1 . Then, the corresponding $\mathrm{SU}$ uplink achievable rate can be identified by utilizing the obtained PDF of the transmit powers at the PUs. The goal of this paper is to investigate the impacts of the power control scheme at SUs and the subcarrier allocation methods at the $\mathrm{SN}$ on the SU uplink achievable 
rate. Therefore, we have not considered the power control at the PUs in this paper, and more sophisticated performance analysis for the networks with power controls at both SUs and PUs is left for future work.

\subsection{Analysis}

Denote the total average achievable rate by $\hat{C} \triangleq \mathbb{E}[C]$. Then, from the fact $\mathbb{E}[q(X)]=q(0)+\int_{0}^{\infty} \frac{\partial q(u)}{\partial u}$ $\mathbb{P}(X \geq u) d u$ for a non-negative and monotonically increasing function $q(\cdot)$, we have

$$
\hat{C}=\sum_{i \in \mathcal{N} \text { s.t. } f_{i} \in \mathcal{F}} \frac{1}{\log 2} \int_{0}^{\infty} \frac{1}{1+u} \mathbb{P}\left(\gamma_{i} \geq u\right) d u .
$$

Note that as each SU adjusts its transmit power based on the distance to its closest PBS, transmit powers at all SUs $\left\{P_{S, \mathbf{x}}\right\}$ are dependent on the locations of PBSs $\Phi_{P}$, and this introduces correlation among the powers $\left\{P_{S, \mathbf{x}}\right\}$ and correlation between $I_{S, i}$ and $I_{P, i}$. For analytical tractability, we assume that the transmit powers at SUs $\left\{P_{S, \mathbf{x}}\right\}$ are independent with each other, and $I_{S, i}$ and $I_{P, i}$ are independent. Thus, we obtain

$$
\begin{aligned}
\mathbb{P} & \left(\gamma_{i} \geq u\right) \\
= & \mathbb{P}\left(h_{o, i} \geq \frac{u\left(I_{S, i}+I_{P, i}+\sigma^{2}\right)\|\mathbf{y}\|^{\alpha_{S}}}{P_{S, o}}\right) \\
\stackrel{(\mathrm{a})}{=} & \mathbb{E}\left[\exp \left(-\frac{u \sigma^{2}\|\mathbf{y}\|^{\alpha_{S}}}{P_{S, o}}\right)\right. \\
& \left.\times \exp \left(-\frac{u\left(I_{S, i}+I_{P, i}\right)\|\mathbf{y}\|^{\alpha_{S}}}{P_{S, o}}\right)\right] \\
= & \int_{0}^{\infty} \mathbb{E}\left[\exp \left(-\frac{u \sigma^{2} r^{\alpha_{S}}}{P_{S, o}}\right) \exp \left(-\frac{u\left(I_{S, i}+I_{P, i}\right) r^{\alpha_{S}}}{P_{S, o}}\right)\right] \\
& \times f_{r}(r) d r \\
\approx & \int_{0}^{\infty} \int_{0}^{\infty} \exp \left(-\frac{u \sigma^{2} r^{\alpha_{S}}}{p}\right) \mathcal{L}_{I_{S, i}}\left(\frac{u r^{\alpha}}{p}\right) \\
& \times \mathcal{L}_{I_{P, i}}\left(\frac{u r^{\alpha_{S}}}{p}\right) f_{P_{S}}(p) f_{r}(r) d p d r,
\end{aligned}
$$

where (a) follows from the fact that the CDF of an exponential random variable $X$ with mean $\rho$ is $\mathbb{P}(X<x)=$ $1-\exp (-x / \rho), f_{r}(r)=2 \pi \lambda_{S} r \exp \left(-\pi \lambda_{S} r^{2}\right)$ is the PDF of the distance from the typical SU and the tagged SBS [22], $f_{P_{S}}(p)$ is the PDF of the transmit powers at SUs in (8) or $(10)$, and $\mathcal{L}_{X}(t) \triangleq \mathbb{E}[\exp (-t X)]$ denotes the Laplace transform of a random variable $X$.

Before deriving analytical expressions for the Laplace transforms $\mathcal{L}_{I_{S, i}}$ and $\mathcal{L}_{I_{P, i},}$, we examine the point processes $\Phi_{S, i}^{(u)}$ in (4) and $\Phi_{P, i}^{(u)}$ in (5). For a PBS, the average number of associated PUs is $\lambda_{P}^{(u)} / \lambda_{P}$. Since each PU utilizes $N_{P}$ subcarriers and each subcarrier is allocated to at most one PU, the intensity of $\Phi_{P, i}^{(u)}$ is

$$
\tilde{\lambda}_{P}^{(u)} \triangleq \min \left(\frac{N_{P} \lambda_{P}^{(u)}}{N}, \lambda_{P}\right) .
$$

For the same reason, when the CSA method is adopted at the SN, the intensity of $\Phi_{S, i}^{(u)}$ can be expressed as

$$
\tilde{\lambda}_{\mathrm{S}, \mathrm{CSA}}^{(u)} \triangleq \min \left(\frac{N_{S} \lambda_{S}^{(u)}}{N}, \lambda_{S}\right) .
$$

On the other hand, when the DSA method is employed at the SN, the intensity of $\Phi_{S, i}^{(u)}$ is equal to

$$
\tilde{\lambda}_{\mathrm{S}, \mathrm{CSA}}^{(u)} \triangleq \frac{N_{S} \lambda_{S}^{(u)}}{N} .
$$

However, since the points in $\Phi_{P, i}^{(u)}\left(\right.$ or $\left.\Phi_{S, i}^{(u)}\right)$ are relevant to the Voronoi tessellation induced by $\Phi_{P}$ (or $\Phi_{S}$ ), the points are correlated, and hence it is intractable to identify the exact distributions of $\Phi_{S, i}^{(u)}$ and $\Phi_{P, i}^{(u)}$. To circumvent this difficulty, we approximate $\Phi_{P, i}^{(u)}$ and $\Phi_{S, i}^{(u)}$ as homogeneous PPPs with intensities $\tilde{\lambda}_{P}^{(u)}$ and $\tilde{\lambda}_{\mathrm{S}, \mathrm{CSA}}^{(u)}\left(\right.$ or $\left.\tilde{\lambda}_{\mathrm{S}, \mathrm{CSA}}^{(u)}\right)$, respectively.

Due to the stationarity of the homogeneous PPP, the Laplace transform $\mathcal{L}_{I_{P, i}}$ can be approximated as

$$
\begin{aligned}
& \mathcal{L}_{I_{P, i}}(t) \\
& =\mathbb{E}\left[\exp \left(-t \sum_{\mathbf{x} \in \Phi_{P, i}^{(u)}} P_{P} g_{\mathbf{x}, i}\|\mathbf{x}\|^{-\alpha_{S}}\right)\right] \\
& \stackrel{(\mathrm{b})}{=} \mathbb{E}_{\Phi_{P, i}}\left[\prod_{\mathbf{x} \in \Phi_{P, i}^{(u)}} \mathbb{E}_{g_{\mathbf{x}, i}}\left[\exp \left(-t P_{P} g_{\mathbf{x}, i}\|\mathbf{x}\|^{\left.-\alpha_{S}\right)}\right]\right]\right. \\
& \stackrel{(\mathrm{c})}{=} \mathbb{E}_{\Phi_{P, i}}\left[\prod_{\mathbf{x} \in \Phi_{P, i}^{(u)}}\left(1+t P_{P}\|\mathbf{x}\|^{-\alpha_{S}}\right)^{-1}\right] \\
& \stackrel{(\mathrm{d})}{\approx} \exp \left(-2 \pi \tilde{\lambda}_{P}^{(u)} \int_{0}^{\infty} \frac{x}{1+x^{\alpha_{S}} /\left(t P_{P}\right)} d x\right) \\
& =\exp \left(-\frac{2 \pi^{2} \tilde{\lambda}_{P}^{(u)}}{\alpha_{S} \sin \left(2 \pi / \alpha_{S}\right)} t^{2 / \alpha_{S}} P_{P}^{2 / \alpha_{S}}\right),
\end{aligned}
$$

where (b) follows from the independence between $\Phi_{P, i}$ and $g_{\mathbf{x}, i},(\mathrm{c})$ comes from the fact that the Laplace transform of an exponential random variable $X$ with mean $\rho$ is $\mathcal{L}_{X}(t)=1 /(1+\rho t)$, and $(\mathrm{d})$ is due to the PPP probability generating functional (PGFL) [22].

Before examining the Laplace transform $\mathcal{L}_{I_{S, i}}(t)$, let us compare $I_{S, i}$ in the cases with the DSA and the CSA. When the DSA is applied, each SU chooses subcarriers independently with respect to other SUs. Thus, as illustrated in Fig. 1, the desired signal may collide not only 




Fig. 1 When the DSA is adopted, there exist interferences from not only other cell SUs but also from intra-cell SUS

with the signals from SUs associated with other SBSs but also with the signals from SUs connected to the tagged SBS. On the other hand, when the CSA is adopted, each SBS assigns orthogonal sets of subcarriers to its associated SUs. Hence, the desired signal does not collide with the signals from other SUs communicating with the tagged SBS as illustrated in Fig. 2.

We would like to mention that, in the previous works in [23-25], each transmitter adjusts its transmit power based on the distance between the transmitter and its intended receiver. Since both transmit power and desired signal term are determined by the distance between a transmitter and its receiver, it is tractable to characterize the performance of the networks in [23-25]. On the other hand, in underlay cognitive radio networks, transmit power at a SU is computed based on the distance between the SU and its nearest PBS, and the desired signal term is relevant to the distance between the SU and its closest SBS. Therefore, analyzing the performance of underlay cognitive radio networks is more challenging than those of the networks in [23-25].

In the following lemma, we derive an approximation of $\mathcal{L}_{I_{S, i}}(t)$ in the case with the CSA where the interfering SUs are located outside of the Voronoi cell of the tagged SBS.
Lemma 2 When the CSA is adopted, the Laplace transform of the interference from other $S U_{s} \mathcal{L}_{I_{S, i}}(t)$ is approximated by

$$
\begin{aligned}
& \mathcal{L}_{I_{S, i}}(t) \\
& \approx \exp \left(-2 \pi \tilde{\lambda}_{\mathrm{S}, \mathrm{CSA}}^{(u)} t^{2 / \alpha_{S}} \eta \int_{r\left(\frac{1}{t p}\right)^{1 / \alpha_{S}}}^{\infty} \frac{y}{1+y^{\alpha S}} d y\right),
\end{aligned}
$$

where $\eta \triangleq \int_{0}^{\infty} q^{2 / \alpha_{S}} f_{P_{S}}(q) d q$ and $f_{P_{S}}(q)$ is the PDF of transmit power $P_{S}$ in (8) or (10).

Proof See Appendix B.

When the DSA is employed, unlike the case with the CSA, the interfering SUs can be located in the Voronoi cell of the tagged SBS. Hence, by changing the lower limit of the integral in (15) to zero and replacing $\tilde{\lambda}_{\mathrm{S}, \mathrm{CSA}}^{(u)}$ with $\tilde{\lambda}_{\mathrm{S}, \mathrm{CSA}}^{(u)}$, an approximation of the Laplace transform $\mathcal{L}_{I_{S, i}}(t)$ can be derived as

$$
\begin{aligned}
\mathcal{L}_{I_{S, i}}(t) & \approx \exp \left(-2 \pi \tilde{\lambda}_{\mathrm{S}, \mathrm{CSA}}^{(u)} t^{2 / \alpha_{S}} \eta \int_{0}^{\infty} \frac{y}{1+y^{\alpha_{S}}} d y\right) \\
& =\exp \left(-\frac{2 \pi^{2} \eta \tilde{\lambda}_{S, D S A}^{(u)}}{\alpha_{S} \sin \left(2 \pi / \alpha_{S}\right)} t^{2 / \alpha_{S}}\right)
\end{aligned}
$$

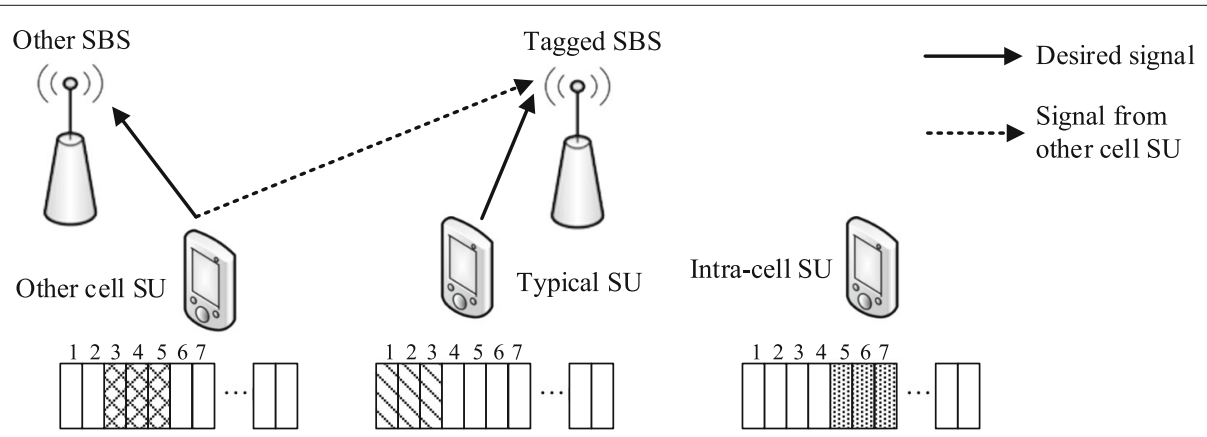

Fig. 2 When the CSA is employed, there are no interferences from intra-cell SUs 
Finally, when the CSA (or DSA) is adopted, we can evaluate $\hat{C}$ in (11) by plugging (12), (14), (15) (or (16)) into (11). From the derived analytical expressions, we can expect that $\hat{C}$ is an increasing function of the number of available subcarriers $N$ since an increase in $N$ leads to decreases in the intensities of interfering users, i.e., $\tilde{\lambda}_{\mathrm{S}, \mathrm{CSA}}^{(u)}, \tilde{\lambda}_{\mathrm{S}, \mathrm{CSA}}^{(u)}$ and $\tilde{\lambda}_{P}^{(u)}$. When $N \rightarrow \infty$, the Laplace transforms $\mathcal{L}_{I_{P, i}}$ and $\mathcal{L}_{I_{S, i}}$ in (12) become one since $\tilde{\lambda}_{P}^{(u)}, \tilde{\lambda}_{\mathrm{S}, \mathrm{CSA}}^{(u)}$, and $\tilde{\lambda}_{\mathrm{S}, \mathrm{CSA}}^{(u)}$ converge to zero. In this case, if $N_{S} / N \rightarrow 0$, an increase of $N_{S}$ leads to an enhanced total average achievable rate $\hat{C}$ as the number of summations in (11) gets larger as $N_{S}$ grows. Also, from the definitions of $\tilde{\lambda}_{\mathrm{S}, \mathrm{CSA}}^{(u)}, \tilde{\lambda}_{\mathrm{S}, \mathrm{CSA}}^{(u)}$, and $\tilde{\lambda}_{P}^{(u)}$, we can infer that $\mathbb{P}\left(\gamma_{i} \geq u\right)$ in (12) decays as $N_{S}$ and $N_{P}$ get larger.

\section{Simulation results}

Next, we present numerical simulation results to validate our analytical results. Unless otherwise stated, we set $\alpha_{P}=\alpha_{S}=4, P_{P}=20 \mathrm{dBm}, \sigma^{2}=-120 \mathrm{dBm}, N=128$, $N_{P}=30, \lambda_{P}^{(u)}=3 \times 10^{-4} / \mathrm{m}^{2}, \lambda_{S}^{(u)}=6 \times 10^{-4} / \mathrm{m}^{2}$, and $P_{\max }=\infty$. Figure 3 examines the CDFs of the transmit power at a SU for different values of $\beta, \lambda_{P}$, and $\tau$. First of all, we observe that our analytical result in (7) is perfectly matched by the simulation results. Since the degree of repulsion among the locations of PBSs gets bigger as $\beta$ increases, the locations of PBSs tend to be distributed more evenly when $\beta$ is large. In this regard, the distance between a SU and its nearest PBS becomes smaller as $\beta$ grows, and thus the transmit power at the SU decreases when $\beta$ becomes higher as shown in Fig. 3. In addition, as expected from (1) and (7), the transmit powers at SUs decay when $\lambda_{P}$ increases and $\tau$ gets smaller. This phenomenon can also be explained by the fact that an increase of $\lambda_{P}$ results in a decrease of the distance between a SU and its closest PBS.

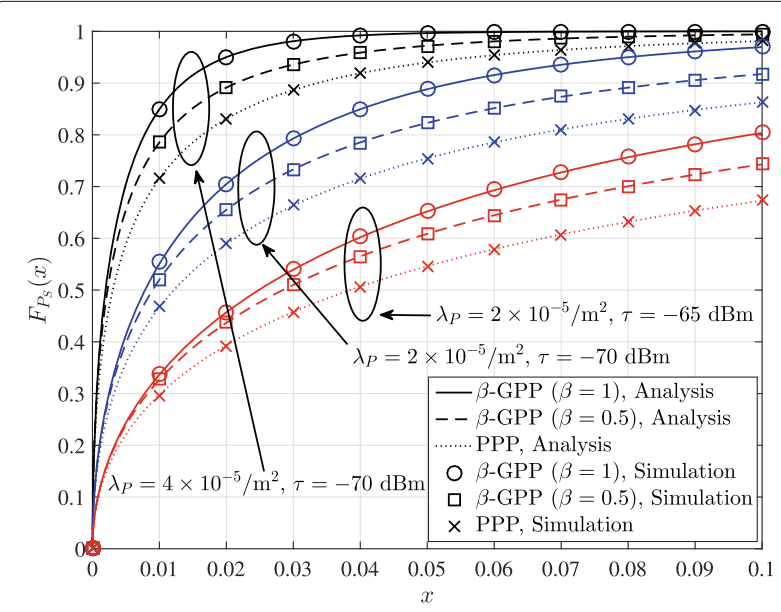

Fig. 3 CDF of transmit powers at SUs
In Fig. 4, we present the total average $\mathrm{SU}$ achievable rate $\hat{C}$ in (11) when $\lambda_{P}=5 \times 10^{-5} / \mathrm{m}^{2}, N_{S}=10$, and the DSA is adopted. From Fig. 4 , we infer that our analytical result predicts well $\hat{C}$. Since a larger $\lambda_{S}$ leads to a smaller distance between a typical SU and its associated SBS, $\hat{C}$ gets higher when $\lambda_{S}$ grows. On the other hand, from (1), the SINR $\gamma_{i}$ (3) is an increasing function of $\tau$, and thus $\hat{C}$ becomes larger when $\tau$ increases as observed in Fig. 4 . Note that, since transmit power at a $\mathrm{SU}$ is a decreasing function of $\beta$, both $P_{S, o}$ and $\left\{P_{S, \mathbf{x}}\right\}$ in (3) get higher as $\beta$ decays, and thus it is difficult to analytically identify the impact of $\beta$ on $\hat{C}$. Instead, under the assumption that the influences of $\beta$ on $P_{S, o}$ and $\left\{P_{S, \mathbf{x}}\right\}$ are the same, we can infer that the SINR $\gamma_{i}$ in (3) will be decreased with $\beta$. From Fig. 4, it is seen that $\hat{C}$ decays as the degree of repulsion among the PBSs grows, i.e., $\beta \rightarrow 1$.

Figure 5 illustrates the total average $\mathrm{SU}$ achievable rate $\hat{C}$ in (11) for different values of $P_{\max }$ and $\alpha_{P}$ when $\lambda_{P}=5 \times$ $10^{-5} / \mathrm{m}^{2}, N_{S}=10$ and the DSA is employed. It is shown that $\hat{C}$ is enhanced when $P_{\max }$ or $\alpha_{P}$ becomes bigger since the transmit powers at SUs are increased with $P_{\max }$ and $\alpha_{P}$. Also, we can observe that $\hat{C}$ is sensitive to $P_{\max }$ when $\alpha_{P}$ is large as the SUs are more likely to use the maximum transmit power $P_{\max }$ when $\alpha_{P}$ is high.

Figure 6 demonstrates the total average SU achievable rate $\hat{C}$ in (11) for the case where $\beta=1, \lambda_{P}=5 \times 10^{-5} / \mathrm{m}^{2}$, and $\lambda_{S}=10^{-4} / \mathrm{m}^{2}$. First, it is shown that $\hat{C}$ is enhanced when the CSA method, which mitigates the interferences from other SUs, is applied at the SN. As the total SU achievable rate $\hat{C}$ is the sum of capacities for each subcarrier as seen in (6), $\hat{C}$ grows as the number of subcarriers for each $\mathrm{SU} N_{S}$ increases. On the other hand, the desired signal is more likely to collide with signals from other SUs as $N_{S}$ becomes higher. Hence, when $N_{S}$ is large, $\hat{C}$ is sensitive to the subcarrier allocation methods, i.e., the gap

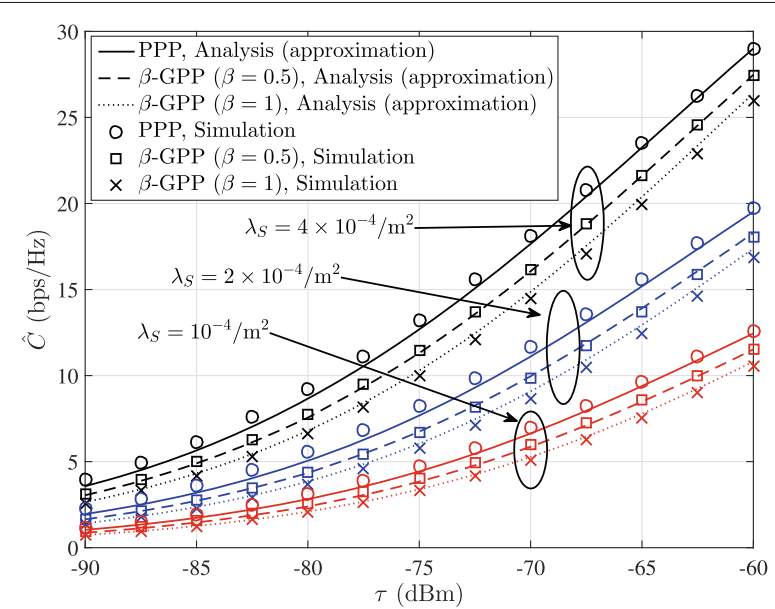

Fig. 4 The total average $S U$ achievable rate $\hat{C}$ as a function of $\tau$ when the DSA is applied at the SN 




Fig. 5 The total average SU achievable rate $\hat{C}$ as a function of $\tau$ when the maximum transmit power constraint is considered

between the capacities with the CSA and the DSA gets bigger as $N_{S}$ grows.

In Fig. 7, we evaluate the total average SU achievable rate $\hat{C}$ in (11) for difference values of $\lambda_{P}$ when $\beta=1, \lambda_{S}=$ $10^{-4} / \mathrm{m}^{2}$, and $N_{S}=10$. From Fig. 7 , we can infer that our analytical results are well matched with the simulated results and the performance is improved when the CSA is adopted at the SN. As observed from Fig. 3, the transmit powers at SUs decay when $\lambda_{P}$ is large. Moreover, since $\tilde{\lambda}_{P}^{(u)}$ in (13) is a non-decreasing function of $\lambda_{P}$, the interference from PUs increases as $\lambda_{P}$ becomes larger. In this regard, the $\mathrm{SU}$ achievable rate $\hat{C}$ gets lower as $\lambda_{P}$ grows.

\section{Conclusions}

This paper studied an underlay OFDM-based CRN where the locations of PBSs are modeled as a $\beta$-GPP. The scenario where the SUs control their transmit powers to

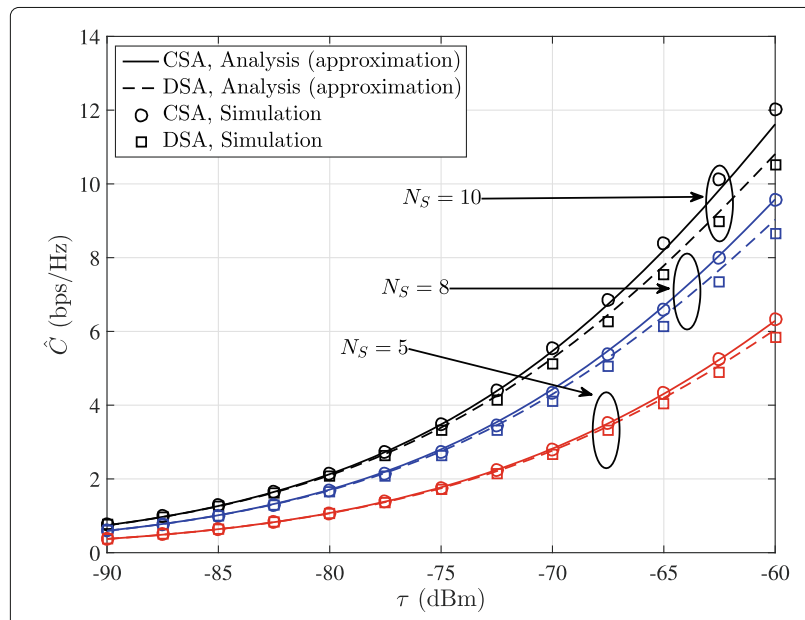

Fig. 6 The total average SU achievable rate $\hat{C}$ as a function of $\tau$ when the spatial distribution of PBSs follows a $\beta$-GPP $(\beta=1)$

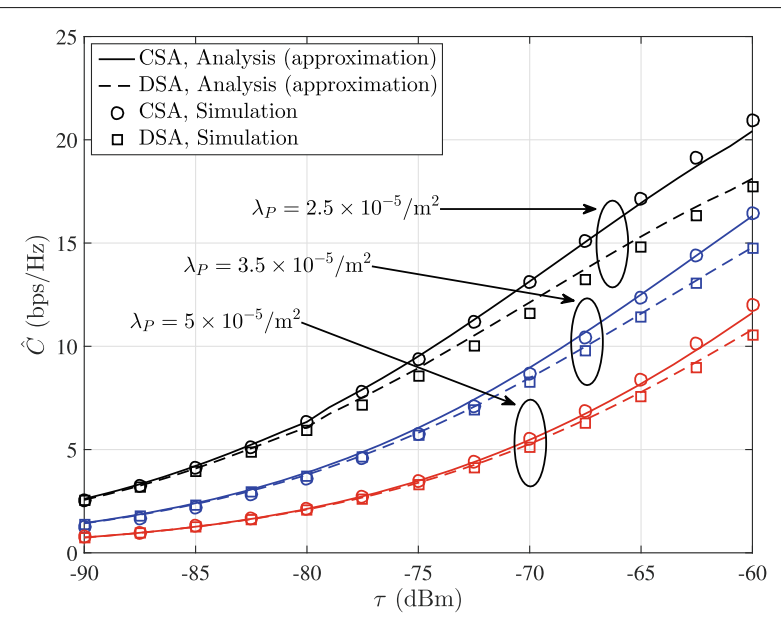

Fig. 7 Impact of the intensity of PBSs $\lambda p$ on the total average SU achievable rate $\hat{C}$

alleviate the inferences at the PBSs is considered. Also, two types of subcarrier allocation techniques are considered depending on whether the subcarriers for the SN are allocated in a centralized manner or not. First, we have identified the characteristics of the transmit powers at SUs. Then, we have derived approximations of the total average $\mathrm{SU}$ achievable rate and verified the tightness of the approximations via numerical simulations. In addition, from simulations, we have observed that the transmit power at SUs and the SU achievable rate become smaller as the degree of repulsion among the PBS increases, and that the SU achievable rate is enhanced when the centralized subcarrier allocation method is applied at the SN.

\section{Appendix A Proof of Lemma 1}

From the definition of $P_{S}$ in (1), the CDF of $P_{S}$ is derived as follows:

$$
\begin{aligned}
F_{P_{S}}(x) & =\mathbb{P}\left(P_{S} \leq x\right) \\
& =\mathbb{P}\left(r_{P} \leq(x / \tau)^{1 / \alpha_{P}}\right) \\
& =1-\mathbb{P}\left(\forall \mathbf{y}_{k} \in \Phi_{P},\left\|\mathbf{y}_{k}\right\|>(x / \tau)^{1 / \alpha_{P}}\right) \\
& =1-\mathbb{P}\left(\forall \mathbf{y}_{k} \in \Phi_{P},\left\|\mathbf{y}_{k}\right\|^{2}>(x / \tau)^{2 / \alpha_{P}}\right) \\
& \stackrel{(\mathrm{e})}{=} 1-\prod_{k=1}^{\infty}\left(\beta \mathbb{P}\left(B_{P, k} \geq(x / \tau)^{2 / \alpha_{P}}\right)+1-\beta\right) \\
& \stackrel{(\mathrm{f})}{=} 1-\prod_{k=1}^{\infty}\left(1-\frac{\beta}{\Gamma(k)} \gamma\left(k, \frac{\pi \lambda_{P} x^{2 / \alpha_{P}}}{\beta \tau^{2 / \alpha_{P}}}\right)\right),
\end{aligned}
$$

where (e) follows from the fundamental property of the $\beta$-GPP and (f) comes from the fact that the CDF of a gamma random variable $X \sim \mathcal{G}(a, b)$ is $\mathbb{P}(X<x)=$ $\gamma(a, x / b) / \Gamma(a)$. 
By differentiating $F_{P_{S}}(x)$ in (17) with respect to $x$, we obtain the PDF of $P_{S}$ :

$$
\begin{aligned}
f_{P_{S}}(x)= & \frac{\partial F_{P_{S}}(x)}{\partial x} \\
= & -\frac{\partial\left(1-F_{P_{S}}(x)\right)}{\partial x} \\
= & -\frac{\partial}{\partial x}\left\{\prod_{k=1}^{\infty} a_{k}(x)\right\} \\
= & -\prod_{k=1}^{\infty} a_{k}(x) \frac{\partial}{\partial x}\left\{\ln \left(\prod_{k=1}^{\infty} a_{k}(x)\right)\right\} \\
= & \frac{2 \beta}{\alpha_{P}} \exp \left(-\frac{\pi \lambda_{P}}{\beta}(x / \tau)^{2 / \alpha_{P}}\right) \prod_{k=1}^{\infty} a_{k}(x) \\
& \times \sum_{k=1}^{\infty} \frac{1}{a_{k}(x) \Gamma(k)}\left(\frac{\pi \lambda_{P}}{\beta \tau^{2 / \alpha_{P}}}\right)^{k} x^{2 k / \alpha_{P}-1},
\end{aligned}
$$

where $a_{k}(x) \triangleq 1-\frac{\beta}{\Gamma(k)} \gamma\left(k, \pi \lambda_{P} x^{2 / \alpha_{P}} /\left(\beta \tau^{2 / \alpha_{P}}\right)\right)$.

\section{Appendix B Proof of Lemma 2}

Note that, in the uplink scenario, $\|\mathbf{y}\|$ is not always smaller than the distances between the tagged SBS and interfering SUs, i.e., $\|\mathbf{x}-\mathbf{y}\|$ for $\mathbf{x} \in \Phi_{S, i}^{(u)} \backslash o$. In addition, since the transmit powers at $\mathrm{SUs}\left(P_{S, o}\right.$ and $\left.P_{S, \mathbf{x}}\right)$ depend on the distribution of PBSs $\Phi_{P}$, the average power of the desired signal $P_{S, o}\|\mathbf{y}\|^{-\alpha_{S}}$ is not always larger than the average interference power $P_{S, \mathbf{x}}\|\mathbf{x}-\mathbf{y}\|^{-\alpha_{S}}$ for $\mathbf{x} \in \Phi_{S, i}^{(u)} \backslash o$. In such a scenario, deriving the exact expression for $\mathcal{L}_{I_{S, i}}(t)$ is intractable. To circumvent this difficulty, we assume that the weighted Voronoi cell of the tagged SBS for the typical SU is a ball with radius $P_{S, o}\|\mathbf{y}\|^{-\alpha_{S}}$, i.e., $P_{S, o}\|\mathbf{y}\|^{-\alpha_{S}}>$ $P_{S, \mathbf{x}}\|\mathbf{x}-\mathbf{y}\|^{-\alpha_{S}}$ for $\mathbf{x} \in \Phi_{S, i}^{(u)} \backslash o$. Then, an approximation of $I_{S, i}$ is expressed as follows:

$$
\begin{aligned}
I_{S, i} \approx & \sum_{\mathbf{x} \in \Phi_{S, i}^{(u)}} P_{S, \mathbf{x}} h_{\mathbf{x}, i}\|\mathbf{x}-\mathbf{y}\|^{-\alpha_{S}} \\
& \times \mathbb{1}\left(P_{S, o}\|\mathbf{y}\|^{-\alpha_{S}}>P_{S, \mathbf{x}}\|\mathbf{x}-\mathbf{y}\|^{-\alpha_{S}}\right) .
\end{aligned}
$$

Our approximation (18) only takes into account the interfering signals whose powers are larger than the power of the desired signal, and thus the approximation can be interpreted as a lower-bound of $I_{S, i}$.

By leveraging the approximation in (18), the following derivations can be established:

$$
\begin{aligned}
& \mathcal{L}_{I_{S, i}}(t) \\
& \stackrel{(\mathrm{g})}{\approx} \mathbb{E}\left[\operatorname { e x p } \left(-t \sum_{\mathbf{x} \in \Phi_{S, i}^{(u)}} P_{S, \mathbf{x}} h_{\mathbf{x}, i}\|\mathbf{x}-\mathbf{y}\|^{-\alpha_{S}}\right.\right. \\
& \left.\left.\times \mathbb{1}\left(p r^{-\alpha_{S}}>P_{S, \mathbf{x}}\|\mathbf{x}-\mathbf{y}\|^{-\alpha_{S}}\right)\right)\right] \\
& \stackrel{(\mathbf{h})}{=} \mathbb{E}\left[\operatorname { e x p } \left(-t \sum_{\mathbf{x} \in \Phi_{S, i}^{(u)}} P_{S, \mathbf{x}} h_{\mathbf{x}, i}\|\mathbf{x}\|^{-\alpha_{S}}\right.\right. \\
& \left.\left.\times \mathbb{1}\left(p r^{-\alpha_{S}}>P_{S, \mathbf{x}}\|\mathbf{x}\|^{-\alpha_{S}}\right)\right)\right] \\
& \stackrel{(\mathrm{i})}{\approx} \mathbb{E}_{\Phi_{S, i}^{(u)}}\left[\prod_{\mathbf{x} \in \Phi_{S, i}^{(u)}}\right. \\
& \left.\times \mathbb{E}_{P_{S, \mathbf{x}}}\left[\frac{1}{1+t P_{S, \mathbf{x}}\|\mathbf{x}\|^{-\alpha_{S} \mathbb{1}\left(p r^{-\alpha_{S}}>P_{S, \mathbf{x}}\|\mathbf{x}\|^{-\alpha_{S}}\right)}}\right]\right] \\
& =\mathbb{E}_{\Phi_{S, i}^{(u)}}\left[\prod_{\mathbf{x} \in \Phi_{S, i}^{(u)}}\right. \\
& \left.\times \int_{0}^{\infty} \frac{1}{1+t q\|\mathbf{x}\|^{-\alpha_{S}} \mathbb{1}\left(p r^{-\alpha_{S}}>q\|\mathbf{x}\|^{-\alpha_{S}}\right)} f_{P_{S}}(q) d q\right] \\
& \stackrel{(j)}{\approx} \exp \left(-2 \pi \tilde{\lambda}_{S, C S A}^{(u)}\right. \\
& \left.\times \int_{0}^{\infty} \int_{0}^{\infty} \frac{x}{1+\frac{x^{\alpha} S}{t q \mathbb{1}\left(p r^{-\alpha}>q x^{-\alpha}\right)}} d x f_{P_{S}}(q) d q\right) \\
& =\exp \left(-2 \pi \tilde{\lambda}_{S, C S A}^{(u)}\right. \\
& \left.\times \int_{0}^{\infty} \int_{r\left(\frac{q}{p}\right)^{1 / \alpha_{S}}}^{\infty} \frac{x}{1+x^{\alpha} /(t q)} d x f_{P_{S}}(q) d q\right) \\
& \stackrel{(\mathrm{k})}{=} \exp \left(-2 \pi \tilde{\lambda}_{S, C S A}^{(u)} t^{2 / \alpha_{S}} \eta \int_{r\left(\frac{1}{t p}\right)^{1 / \alpha_{S}}}^{\infty} \frac{y}{1+y^{\alpha} S} d y\right) \text {, }
\end{aligned}
$$

where (g), (h), and (i) follow from the approximation in (18), the stationarity of the homogeneous PPP and the assumption that the transmit powers $\left\{P_{S, \mathbf{x}}\right\}$ are independent, respectively. Also, (j) and (k) are due respectively to the PGFL of the homogeneous PPP and the variable change $y=x(t q)^{-1 / \alpha_{S}}$, where $\eta \triangleq \int_{0}^{\infty} q^{2 / \alpha_{S}} f_{P_{S}}(q) d q$.

\section{Endnote}

${ }^{1}$ In this paper, non-random $N_{P}$ and $N_{S}$ are considered for analytical tractability. An extension to networks with randomly varying $N_{P}$ and $N_{S}$ is outside the scope of this paper and represents an interesting problem. 


\section{Abbreviations}

AWGN: Additive white Gaussian noise; CDF: Cumulative distribution function: CRN: Cognitive radio networks; CSA: Centralized subcarrier allocation; DSA: Decentralized subcarrier allocation; GPP: Ginibre point process; OFDM: Orthogonal frequency division multiplexing; PBS: Primary base station; PDF: Probability density function; PGFL: Probability generating functional; PN: Primary network; PPP: Poisson point process; PU: Primary user; SBS: Secondary base station; SINR: Signal-to-interference-plus-noise ratio; SN: Secondary network; SU: Secondary user

\section{Acknowledgements}

This work was supported by NSF EARS Award No. 1547447.

\section{Funding}

This work was supported by NSF EARS Award No. 1547447.

\section{Authors' contributions}

$\mathrm{ES}$ and $\mathrm{KQ}$ proposed the conception of subcarrier allocation methods of the study. HBK and SE contributed to the analysis and simulation. All authors read and approved the final manuscript.

\section{Competing interests}

The authors declare that they have no competing interests.

\section{Publisher's Note}

Springer Nature remains neutral with regard to jurisdictional claims in published maps and institutional affiliations.

\section{Author details}

${ }^{1}$ Department of Electrical and Computer Engineering, Texas A\&M University, College Station 77843, TX, USA. ${ }^{2}$ School of Electrical and Computer Engineering, Oklahoma State University, Stillwater 74078, OK, USA.

${ }^{3}$ Department of Electrical and Computer Engineering, Texas A\&M University at Qatar, 23874 Doha, Qatar.

Received: 1 October 2018 Accepted: 4 January 2019

Published online: 24 January 2019

\section{References}

1. H. A. Mahmoud, T. Yucek, H. Arslan, OFDM for cognitive radio: Merits and challenges. IEEE Wireless Commun. 16(2), 6-15 (2009)

2. D. C. Popescu, D. R. Joshi, O. A. Dobre, in Proc. IEEE Asilomar Conf. Signals, Syst., Comput. Spectrum allocation and power control in OFDM-based cognitive radios with target SINR constraints (IEEE, Pacific Grove, 2010)

3. J. Yuan, M. Torlak, in Proc. IEEE Int. Wireless Commun. Mobile Comput. Conf. (IWCMC). Optimization of throughput and autonomous sensing in random access cognitive radio networks (IEEE, Istanbul, 2011)

4. S. Ekin, M. M. Abdallah, K. A. Qaraqe, E. Serpedin, Random subcarrier allocation in OFDM-based cognitive radio networks. IEEE Trans. Signal Process. 60(9), 4758-4774 (2012)

5. S. Ekin, M. M. Abdallah, K. A. Qaraqe, E. Serpedin, A study on inter-cell subcarrier collisions due to random access in OFDM-based cognitive radio networks. IEEE Trans. Commun. 61(5), 1695-1707 (2013)

6. G. A. S. Sidhu, F. Gao, W. Wang, W. Chen, Resource allocation in relay-aided OFDM cognitive radio networks. IEEE Trans. Veh. Technol. 62(8), 3700-3710 (2013)

7. D. Darsena, G. Gelli, F. Verde, An opportunistic spectrum access scheme for multicarrier cognitive sensor networks. IEEE Sensors J. 17(8), 2596-2606 (2017)

8. H. A. Shah, I. Koo, A novel physical layer security scheme in OFDM-based cognitive radio networks. IEEE Access. 6, 29486-29498 (2018)

9. M. G. Khoshkholgh, N. M. Yamchi, K. Navaie, H. Yanikomeroglu, V. C. M. Leung, K. G. Shin, Radio resource allocation for OFDM-based dynamic spectrum sharing: Duality gap and time averaging. IEEE J. Sel. Areas Commun. 33(5), 848-864 (2015)

10. M. Chami, M. Pischella, D. L. Ruyet, Resource allocation for OFDM-based multiuser cooperative underlay cognitive systems. EURASIP J. Wirel. Commun. Netw. 2017(1), 180 (2017)

11. H. ElSawy, E. Hossain, M. Haenggi, Stochastic geometry for modeling, analysis, and design of multi-tier and cognitive cellular wireless networks: A survey. IEEE Commun. Surv. Tuts. 15(3), 996-1019 (2013)
12. C.-H. Lee, M. Haenggi, Interference and outage in Poisson cognitive networks. IEEE Trans. Wirel. Commun. 11(4), 1392-1401 (2012)

13. G. Yuchen, N. Kai, L. Jiaru, Stochastic geometric analysis of the uplink throughput in cognitive radio cellular networks. China Commun. 10(8), 44-53 (2013)

14. S. R. Sabuj, M. Hamamura, Uplink modeling of cognitive radio network using stochastic geometry. Perform. Eval. 117, 1-15 (2017)

15. S. Weber, J. G. Andrews, N. Jindal, An overview of the transmission capacity of wireless networks. IEEE Trans. Commun. 58, 3593-3604 (2010)

16. A. Guo, M. Haenggi, Spatial stochastic models and metrics for the structure of base stations in cellular networks. IEEE Trans. Wirel. Commun. 12(11), 5800-5812 (2013)

17. S.-R. Cho, W. Choi, Energy-efficient repulsive cell activation for heterogeneous cellular networks. IEEE J. Sel. Areas Commun. 31, 870-882 (2013)

18. A. Goldman, The Palm measure and the Voronoi tessellation for the Ginibre process. Ann. Appl. Probab. 20(1), 90-128 (2010)

19. N. Miyoshi, T. Shirai, Cellular networks with $\alpha$-Ginibre configurated base stations. The Impact of Applications on Mathematics. (Springer, New York, 2014), pp. 211-226

20. N. Deng, W. Zhou, M. Haenggi, The Ginibre point process as a model for wireless networks with repulsion. IEEE Trans. Wirel. Commun. 14(1), 107-121 (2015)

21. I. Nakata, N. Miyoshi, Spatial stochastic models for analysis of heterogeneous cellular networks with repulsively deployed base stations. Perform. Eval. 78, 7-17 (2014)

22. M. Haenggi, Stochastic geometry for wireless networks. (Cambridge Univ. Press, Cambridge, 2012)

23. H. ElSawy, E. Hossain, On stochastic geometry modeling of cellular uplink transmission with truncated channel inversion power control. IEEE Trans. Wirel. Commun. 13, 4454-4469 (2014)

24. H. ElSawy, A. Sultan-Salem, M.-S. Alouini, M. Z. Win, Modeling and analysis of cellular networks using stochastic geometry: A tutorial. IEEE Commun. Surv. Tuts. 19, 167-203 (2017)

25. T. D. Novlan, H. S. Dhillon, J. G. Andrews, Analytical modeling of uplink cellular networks. IEEE Trans. Wirel. Commun. 12, 2669-2679 (2013)

\section{Submit your manuscript to a SpringerOpen ${ }^{\circ}$ journal and benefit from:}

- Convenient online submission

Rigorous peer review

- Open access: articles freely available online

- High visibility within the field

- Retaining the copyright to your article

Submit your next manuscript at $>$ springeropen.com 